\title{
Etude des facteurs de la satisfaction des usagers du Service de Chirurgie Générale du CHU Gabriel Touré à Bamako
}

\section{Factors associated to the General Surgery Department users' satisfaction, Gabriel Toure University Hospital, Bamako}

Diallo $\mathrm{B}^{1^{*}}$, Kané ATS ${ }^{2}$, Aoudou $\mathrm{H}^{2}$, Sangho $\mathrm{O}^{3}$, Diarra $\mathrm{S}^{3}$, Sanogo $\mathrm{A}^{4}$, Coulibaly $\mathrm{B}^{1}$, Touré $\mathrm{K}^{1}, \mathrm{Ba} \mathrm{B}^{1}$, Diawara $\mathrm{O}^{1}$,

Traoré $\mathrm{H}^{1}$, Traoré $\mathrm{Y}^{4}$, Touré $\mathrm{O}^{4}$, Traoré $\mathrm{A}^{4}$, Nour $\mathrm{MAB}^{5}$, NGatil $\mathrm{M}^{5}$, Nasrasdine $\mathrm{M}^{4}$, Kéita ${ }^{6}$, Keita $\mathrm{H}^{7}$, Sangho $A^{8}$, Touré $F^{9}$, Telly $N^{11}$, Sangho $F^{3}$, Coulibaly $\mathrm{CA}^{11}$, Kéïta $\mathrm{M}^{10}$, Koné $\mathrm{MO}^{11}$

\section{DOI : 10.53318/msp.v11i1.1892}

${ }^{1}$ Centre Hospitalier Universitaire d'Odontostomatologie CHU-OS

2Direction Centrale des Services de Santé des Armées DCSSA

3Département d'Enseignement et de Recherche des Sciences Biologiques et Médicales (DERSBM), Faculté de Pharmacie (FAPH), Université des Sciences, des Techniques et des Technologies de Bamako (USTTB)

4Institut National de Formation en Sciences de la Santé

${ }^{5}$ Centre Hospitalier Universitaire de Référence Nationale de N'Djamena au Tchad

6Institut Universitaire de Gestion

'Direction régionale de la santé de Kidal

${ }^{8}$ Département d'Enseignement et de Recherche des Sciences Pharmaceutiques, FAPH, USTTB

PProgramme National de Lutte contre le Paludisme (PNLP), Bamako, Mali

${ }^{10}$ Centre de Santé de Référence de la Commune 6, Bamako, Mali

${ }^{11}$ Institut National de Santé Publique (INSP), Bamako, Mali

*Correspondant : Dr Baba Diallo ,Hospitalier au CHU CNOS Tel : 74565428, babasidikidiallo@yahoo.fr

\section{RESUME}

Introduction : La satisfaction des usagers est l'un des critères de succès les plus importants et un atout majeur pour le développement d'une bonne relation entre un usager et le médecin. Elle est une composante importante de la qualité des soins. Le patient est considéré comme un client. L'objectif était d'étudier les facteurs influençant la satisfaction des usagers du service de chirurgie générale du CHU-Gabriel Touré. Matériel et méthodes : Nous avons mené une étude transversale descriptive. La méthode d'échantillonnage probabiliste et la technique de sondage systématique ont été utilisées pour sélectionner l'échantillon. L'étude a concerné 80 patients. Le questionnaire était l'outil de collecte des données et le consentement verbal a été obtenu. Résultats : II en ressort que $55 \%$ des répondants sont de sexe féminin, soit un sexe ratio de 1,22 en faveur des femmes. La tranche d'âge la plus représentée était $30-44$ ans avec $32,5 \%$ et une moyenne de $43,71 \pm 15,35$ ans. Nous avons enquêté 62 patients hospitalisés et 18 vus en ambulatoire. Les mariés représentaient $73,8 \%$ des enquêtés. Le taux de satisfaction était de $82,50 \%$. II existait un lien significatif entre le statut matrimonial et la satisfaction globale des usagers $p=0,009$ et entre la propreté de la salle et la satisfaction globale des usagers $p=0,038$. Conclusion : La mesure de la satisfaction des patients est aujourd'hui une préoccupation générale de tous les établissements de santé. La mesure de la satisfaction des patients constitue une source d'informations pour l'amélioration de la qualité de soins.

Mots clés : satisfaction, facteur, usager, chirurgie, Hôpital, Bamako.

\section{ABSTRACT}

Introduction: User satisfaction is one of the most important success criteria and a major asset for the development of a good relationship between a user and the doctor. It is an important component of the quality of care. The patient is considered a client. The objective was to study the factors influencing the satisfaction of users of the general surgery department of the CHU-Gabriel Touré. Materials and methods: We carried out a descriptive cross-sectional study. The probability sampling method and the systematic sampling technique were used to select the sample. The study involved 80 patients. The questionnaire was the data collection tool and verbal consent was obtained. Results: It emerges that $55 \%$ of respondents are female, ie a sex ratio of 1.22 in favor of women. The most represented age group was $30-44$ years with $32.5 \%$ and an average of $43.71 \pm 15.35$ years. We surveyed 62 hospitalized patients and 18 seen on an outpatient basis. The married represented $73.8 \%$ of the respondents. The satisfaction rate was $82.50 \%$. There was a significant link between marital status and overall user satisfaction $p=0.009$ and between room cleanliness and overall user satisfaction $p=0.038$. Conclusion: Measuring patient satisfaction is a general concern of all healthcare establishments today. Measuring patient satisfaction is a source of information for improving the quality of care.

Key words: satisfaction, factor, user, surgery, Hospital, Bamako.

\section{INTRODUCTION}

La satisfaction des usagers est l'action de satisfaire, de combler un besoin, un désir, une demande [1]. Elle est l'un des critères de succès les plus importants et un atout majeur pour le développement d'une bonne relation entre un usager et le médecin [1]. La recherche constante de cette la satisfaction a conduit à beaucoup d'étude à travers le monde [2-10]. Une étude menée en France au $\mathrm{CHU}$ de Sens par Renaut en 2000 notait que $91 \%$ des patients étaient satisfaits de leur hospitalisation [11]. A Djeddah le niveau de satisfaction des usagers de la Chirurgie Générale était de 93,8\% [2]. Pour Thi : « Le malade n'est plus un consultant ou un usager mais un client, au même 
titre que sa famille ou son entourage [12]. Et ce client est devenu plus exigeant quant à la qualité de service [12]. II est plus attentif à l'accueil, à la durée de l'attente, au manque d'informations [12]. II considère la santé comme un droit, un service dû à l'importance de ses cotisations sociales le pousse à avoir un œil critique [12]. La qualité de l'accueil et des relations interpersonnelles impactent la satisfaction des usages du service de chirurgie [5].

Selon Yameogo et al. en 2017, la satisfaction des patients est une composante importante de l'évaluation de la qualité des soins [13]. Au Burkina Faso, une étude menée par Yaméogo en 2017 dans l'unité de Cardiologie a montré que le score global de satisfaction des patients pris en charge dans l'unité d'hospitalisation dans le service de cardiologie était de 78,3\% [13]. Au Niger, Adamou et al. en 2017 ont mené une étude à Zinder et sont parvenus à une satisfaction globale de $54,63 \%$ auprès de 227 patients au Service des Urgences Chirurgicales à I'hôpital National [14]. Au Cameroun Njong en 2020 a évalué la satisfaction des usagers vis-à-vis de la qualité de la santé et les résultats ont montré que plus de $85 \%$ des usagers étaient satisfaits de la qualité globale des services de santé dans le pays [15].

Au Mali, le niveau de satisfaction globale des usagers a fait l'objet d'une évaluation par l'Agence Nationale d'Evaluation des Hôpitaux (ANEH) 2018 au Centre Ho Gabriel Touré, ce taux était de $47 \%$ [16]. Dans le but de contribuer à l'amélioration de la fréquentation de l'hôpital, nous avons décidé mener cette étude. L'objectif était d'étudier les facteurs influençant la satisfaction des usagers du service de chirurgie générale du CHU-GT.

\section{MATERIEL ET METHODES}

II s'agissait d'une enquête transversale descriptive qui s'est déroulée pendant une période d'un mois (du 13 novembre au 13 décembre 2020) dans le service de chirurgie générale du CHU-Gabriel Touré. Etaient inclus dans cette étude les usagers (ambulatoires ou hospitalisés) et les accompagnants des patients qui n'étaient pas capable de répondre au questionnaire. Les patients d'autres services de l'hôpital n'étaient pas concernés par cette étude.

La taille de l'échantillon était de 80 usagers du service de chirurgie générale du CHU-Gabriel Touré CHU. Ces patients ont été sélectionnés en utilisant la technique de sondage aléatoire systématique. Pour avoir le pas de sondage, nous avons divisé 262 (base de sondage $=\mathrm{N}$ ) par 80 (taille de l'échantillon $=n$ ). Pas de sondage $=\mathrm{N} / \mathrm{n}$ $262 / 80=3,28$ (pas de sondage= 3 ). Nous avons tiré au hasard un chiffre entre 1 et le pas de sondage (3). Ce chiffre correspondait à 1. Le premier patient correspondait à notre première personne enquêtée. Toutes les autres unités statistiques ont été tirées en ajoutant le pas de sondage. Le questionnaire a été utilisé comme outil de collecte. II a été adressé aux patients hospitalisés et en ambulatoire selon nos critères d'inclusion. Cependant, les questions sur l'éclairage des salles d'hospitalisation, l'état des toilettes, la disponibilité du personnel de santé en cas de besoin urgent, le temps mis par le personnel en cas d'appel, l'appréciation des matelas et le coût des interventions chirurgicales ont été adressé aux patients hospitalisés uniquement. Les données ont été saisies et analysées avec le logiciel IBM_SPSS 21.0. Les résultats ont été présentés sous forme de tableaux et figures. Nous avons utilisé le test de Khi-deux ou le test exact de Fisher pour tester la relation entre certains variables au seuil de significativité de 0,05.

La fiche de consentement éclairée a été obtenue auprès de chaque personne enquêtée.

\section{RESULTATS}

Les femmes représentaient $55 \%$ des répondants soit un sex ratio de 1,22.

La tranche d'âge 30-44 ans était la plus représentée avec $32,5 \%$ des cas (Tableau I). L'âge moyen était de 43,71 \pm 5 ans (Tableau I).

Les mariés constituaient la majorité des répondants avec $73,8 \%$.

Parmi les usagers, les non scolarisés étaient les plus représentés avec $41,3 \%$ des cas (Tableau II).

Sur les 80 usagers, les patients hospitalisés étaient au nombre de 62 soit $(77,50 \%)$ et les ambulatoires 18 soit $(22,50 \%)$.

Parmi les 62 hospitalisés, $36(58,06 \%)$ estimaient que l'état de la salle d'hospitalisation était bon (Graphique 1). Les hospitalisés trouvaient que l'éclairage des salles était très bon, bon et assez-bon avec respectivement $54,84 \%$ (34/62), $41,94 \%(26 / 62)$ et $3,22 \%(2 / 62)$.

Environ $77,42 \%)$ estimaient que l'état des toilettes était mauvais (Tableau III).

L'appréciation de l'état des matelas par les patients hospitalisés était confortable, assez confortable et mauvais avec respectivement $50 \%$ (31/62), 37,10\% (23/62) et $12,90 \%(8 / 62)$.

Environ $55 \%$ des répondants avaient déclaré que l'accueil était bon (Tableau IV).

Les enquêtés ont signalé que le personnel soignant était disponible dans $97,5 \%$ des cas lorsqu'ils les appelaient. Sur les 62, 85,49\% (53/62) estimaient le mis par le personnel soignant après l'appel en cas d'urgence entre $5-10 \mathrm{mn}, 6,45 \%$ ente $15-20 \mathrm{~min}$ et $8,06 \%$ à $30 \mathrm{~min}$ et plus. La majorité des répondants $91,25 \%$ (73/80) avaient reçu les informations sur leur diagnostic.

Les $3 / 4$ des répondants étaient informés sur l'évolution de leurs maladies.

Moins de la moitié des usagers (45\%) trouvaient que le coût des examens était très cher (Tableau V). Le coût moyen des examens paracliniques était de $50000 \pm 6000$ F CFA ( $77 \pm 9$ euros).

Les coûts des interventions étaient jugé très élevés par $72,59 \%$ des usagers, abordable par $19,35 \%$, les $8,06 \%$ restant n'avait pas d'appréciation. Le coût moyen des interventions chirurgicales était de $70000 \pm 9000$ F CFA (108 \pm 14 euros) 
La satisfaction des usagers du service de chirurgie général du CHU-Gabriel était bonne dans $82,50 \%$ et mauvais dans $17,50 \%$.

Au total $40,0 \%$ des patients jugeaient que le personnel soignant a une très bonne attitude.

Il existait un lien significatif entre le statut matrimonial et la satisfaction globale des usagers, $\mathbf{p}=\mathbf{0 , 0 0 9}$ (Tableau VI). II existait une relation statistiquement significative entre la propreté de la salle et la satisfaction globale des usagers, $p=0,038$ (Tableau VII).

II existait une association statistiquement significative entre la satisfaction globale des usagers et l'état des toilettes, $p=0,002$ (Tableau VIII).

\section{DISCUSSION}

\section{Caractéristiques sociodémographiques des répondants}

Plus de la moitié des enquêtés étaient de sexe féminin. Cette situation pourrait s'expliquerai par le fait que d'une manière générale, en Afrique les femmes consomment plus de soins de santé que les hommes [17].

L'âge moyen est de 43,71 ans \pm 5 ans. Cela signifierait que le service de chirurgie générale est beaucoup plus fréquenté par une population relativement jeune. Cette situation pourrait s'expliquer par les motifs de consultations ou d'hospitalisation en service de chirurgie générale dans nos pays qui portent sur les traumatismes ou les accidents de la voie publique qui concernent plus les populations les jeunes. Zendjidjian et al. trouvent une association entre la satisfaction et les caractéristiques sociodémographiques notamment l'âge [7].

\section{La satisfaction des usagers selon les facteurs} environnementaux hospitaliers

Le taux de satisfaction globale était de $82,50 \%$. Ce résultat est supérieur de celui obtenu par de DIPAMA.D $(41,2 \%)$ [18]. II y avait un lien significatif entre la propriété des salles et la satisfaction globale des patients, $p=0,038$. La satisfaction était de $93,8 \%$ à Djeddah [2]. Nos résultats sont similaires à ceux de NANA W.F. et al. en 2014, dans leur enquête sur la satisfaction des usagers du centre hospitalier régional de Koudougou au Burkina Faso, montrant que la propreté de l'hôpital est satisfaisante pour $86,9 \%$ des usagers [19].

Satisfaction des usagers selon les facteurs liés aux personnels soignants

Plus du tiers des patients jugeaient que le personnel soignant $a$ une très bonne attitude. Selon ZIDA E. et al. en 2013 , seulement $11,67 \%$ des agents ont de l'empathie envers les patients et $27,78 \%$ des agents ont une écoute attentive envers les patients. Cette insuffisance dans la qualité humaine de l'accueil est source de conflits et de désertions des services de santé au profit d'autres structures sanitaires [20].

\section{La Satisfaction des usagers selon les facteurs liés aux} soins

Nos résultats sur l'appréciation des coûts des examens sont similaires à ceux de NANA W.F.et al. en 2014 qui trouvent que $58,5 \%$ des patients sont « non satisfaits » du coût des examens. Pour le coût des examens seulement $15 \%$ et $28 \%$ aux urgences médicales et en pédiatrie sont satisfaits [19]. La satisfaction lié aux soins prodigués a été notée par Zendjidjian et al., Schoenfelder et al. et Amorim et al. $[5,7,8]$. Nos résultats sont aussi proches de ceux de Kamelia A. et al. au Maroc, chez qui $67,2 \%$ des patients atteints de cancer dans un hôpital universitaire, trouvent le coût du séjour très cher pour des enquêtés [21]

Concernant le délai d'attente avant la prise en charge, les résultats de notre étude, montrent que près de la moitié des enquêtés ont attendu plus de $30 \mathrm{mn}$ avant d'être pris en charge par un soignant. Ce long temps d'attente constitue des pressions psychologiques pour les patients et les accompagnants et sont sources d'insatisfactions comme le montre l'étude de Abidova et al. [6]. Certaines études ont identifié la liaison entre la satisfaction et la nature et durée des souffrances en ce sens que plus le patient dure dans la structure moins il y a de satisfaction $[3,4]$.

\section{CONCLUSION}

Les enquêtés étaient globalement satisfaits concernant l'informations par le personnel de santé sur le diagnostic, l'évolution de la maladie. L'indentification des facteurs de la satisfaction des usagers contribue à améliorer la qualité des soins dans nos structures de santé. Pour mieux satisfaire les usagers dans le service de chirurgie générale du CHU-Gabriel Touré, il est nécessaire d'améliorer l'hygiène des toilettes et des salles d'hospitalisation.

\section{REFERENCES}

1. Hamilton DF, Lane JV, Gaston P, Patton JT, Macdonald D, Simpson AHRW, et al. What determines patient satisfaction with surgery? A prospective cohort study of 4709 patients following total joint replacement. BMJ Open. 2013;3(4):e002525

2. Almehman BA, Mikwar Z, Balkhy A, Jabali H, Hariri BS, Baatiah NY. Measuring Patient Satisfaction and Factors Affecting it in the General Surgery Setting in Jeddah. Cureus [Internet]. 28 déc 2019 [cité 23 juill 2021];11(12). Disponible sur:

https://www.cureus.com/articles/26127-measuringpatient-satisfaction-and-factors-affecting-it-in-thegeneral-surgery-setting-in-jeddah

3. Donate-Manzanares M, Rodríguez-Cano T, GómezSalgado J, Rodríguez-Almagro J, HernándezMartínez A, Barrilero-Fernández E, et al. Quality of Childbirth Care in Women Undergoing Labour: Satisfaction with Care Received and How It Changes over Time. J Clin Med. 29 mars 2019;8(4):434.

4. Choi I, Kim JK, Kim SJ, Cho SC, Kim IN. Satisfaction Levels and Factors Influencing Satisfaction With Use of a Social App for Neonatal and Pediatric Patient Transfer Information Systems: A Questionnaire Study Among Doctors. JMIR Med Inform. 4 août 2016;4(3):e26. 
5. Schoenfelder T, Klewer J, Kugler J. Factors Associated with Patient Satisfaction in Surgery: The Role of Patients' Perceptions of Received Care, Visit Characteristics, and Demographic Variables. J Surg Res. 1 nov 2010;164(1):e53-9.

6. Abidova A, da Silva PA, Moreira S. The mediating role of patient satisfaction and perceived quality of healthcare in the emergency department. Medicine (Baltimore). 19 mars 2021;100(11):e25133.

7. Zendjidjian XY, Auquier $P$, Lançon C, Loundou A, Parola N, Faugère $M$, et al. Determinants of patient satisfaction with hospital health care in psychiatry: results based on the SATISPSY-22 questionnaire. Patient Prefer Adherence. 24 oct 2014:8:1457-64.

8. Amorim L de $P$, Senna MIB, Alencar GP, Rodrigues LG, de Paula JS, Ferreira RC. User satisfaction with public oral health services in the Brazilian Unified Health System. BMC Oral Health. 25 juin 2019:19:126.

9. Song E-O, Jang $\mathrm{H}-\mathrm{Y}$. Predictors of Satisfaction with Care Services among Family Members of Older Adult Residents of Long-Term Care Facilities. Int J Environ Res Public Health. mai 2020;17(9):3298.

10. Orlando JF, Beard M, Kumar S. Systematic review of patient and caregivers' satisfaction with telehealth videoconferencing as a mode of service delivery in managing patients' health. PLoS ONE. 30 août 2019;14(8):e0221848.

11. Renaut $L$. Etude de la satisfaction des usagers : de la mesure de satisfaction à l'amélioration de la qualité au centre hospitalier de Sens (France). 1999 Mémoire Ecole nationale de la santé publique.

12. Thi , Phi, Linh. Nguyen., Satisfaction des patients hospitalisés en France et au Viet Nam 2003 ; Doctoral dissertation, Université Henri Poincaré-Nancy 1.

13. Yameogo AR, Millogo GRC, Palm AF, Bamouni J, Mandi GD, Kologo JK, et al. Évaluation de la satisfaction des patients dans le service de cardiologie du CHU Yalgado Ouedraogo. Pan Afr Med J [Internet]. 27 nov 2017 [cité 23 juill 2021];28(267). Disponible sur: https://www.panafrican-medjournal.com/content/article/28/267/full

14. Adamou, H., Magagi, I. A., Habou, O., Halidou, M., Karimou, S., Sani, R., \&Abarchi, H. Satisfaction des patients, élément important dans la globalité des soins : cas d'un service des urgences chirurgicales au Niger. 2017 ; Mali Médical, 32(2).

15. Njong, A. M., \&Tchouapi, R. P. M Évaluation de la Satisfaction des Usagers Vis-À-Vis de la Qualité des Services de Santé au Cameroun. 2020; Africa Portal $\mathrm{N}^{\circ} 672$

16. Nationale d'Accréditation et d'Évaluation en Santé : La satisfaction des patients lors de leur prise en charge dans les établissements de santé - Revue de la littérature médicale. Sept 1996. Service d'Évaluation Hospitalière. $35 \mathrm{p}$.

17. Centre de recherche, d'étude et de documentation en économie de la santé (CREDES), 2002, Question d'économie de la santé, bulletin d'information en économie de la santé $n^{\circ} 053$ juin 2002.

18. Dipama. D; Nazinigouba Ag, Evaluation de la satisfaction des patients sur la prise en charge dans le service des urgences médicales du centre hospitalier universitaire (CHUYO) ; thèse de médecine, université de Ouagadougou ,2011. $\mathrm{N}^{\circ} 41$, 103 pages

19. Nana.w.f., s. Meda, h hervé, p. Poda, m. K. Drabo. Enquête de satisfaction des usagers du centre hospitalier régional de Koudougou au Burkina Faso. 2018 Revue Bio-Africa - № 18 -, pp. 30-35.

20. Zida e ; ouedraogo s. E. ; a. Zongo Etude des facteurs qui limitent la qualité de l'accueil dans un service des urgences médicales : cas du service des urgences médicales du centre hospitalier universitaire Yalgado OUEDRAOGO, Mémoire de fin d'études 2013, 99 pages

21. Kamelia A, Imane Toughrai, NajibBenmansour, Said Ait Laalim, Mohamed El Amine El Alami, Khalid Mazaz Enquête de satisfaction des patients atteints de cancer dans un hôpital universitaire au Maroc 2013, JournalVol. 25 | pages 627 à 632 (article).

\section{Liste des tableaux et figures}

Tableau I : Répartition des usagers selon la tranche d'âge

\begin{tabular}{lcc}
\hline Tranche d'âge $^{*}$ & Effectif & $\%$ \\
\hline $15-29$ ans & 18 & 22,5 \\
$30-44$ ans & 26 & 32,5 \\
$45-59$ ans & 21 & 26,3 \\
$60-74$ ans & 15 & 18,8 \\
Total & $\mathbf{8 0}$ & 100,0 \\
\hline
\end{tabular}

*'âge moyen était de 43,71 \pm 5 ans.

Tableau III : Répartition des usagers selon leur niveau d'instruction

\begin{tabular}{lcc}
\hline Niveau d'instruction & Effectif & $\%$ \\
\hline Aucun & 33 & 41,3 \\
Primaire & 17 & 21,3 \\
Secondaire & 16 & 20,0 \\
Supérieur & 14 & 17,5 \\
Total & $\mathbf{8 0}$ & $\mathbf{1 0 0 , 0}$ \\
\hline
\end{tabular}

Tableau III : Répartition des usagers selon l'appréciation de l'état des toilettes

\begin{tabular}{lrr}
\hline Etat des toilettes & Effectif & $\%$ \\
\hline Très bon & 1 & 1,61 \\
Bon & 8 & 12,90
\end{tabular}




\begin{tabular}{lrr} 
Assez- bon & 3 & 4,84 \\
Mauvais & 48 & 77,42 \\
Ne sait pas & 2 & 3,23 \\
Total & 62 & 100,00 \\
\hline
\end{tabular}

Tableau IV : Répartition des usagers selon l'appréciation de l'accueil

\begin{tabular}{l}
$\begin{array}{l}\text { Accueil du personnel } \\
\text { soignant }\end{array}$ \\
\hline
\end{tabular}

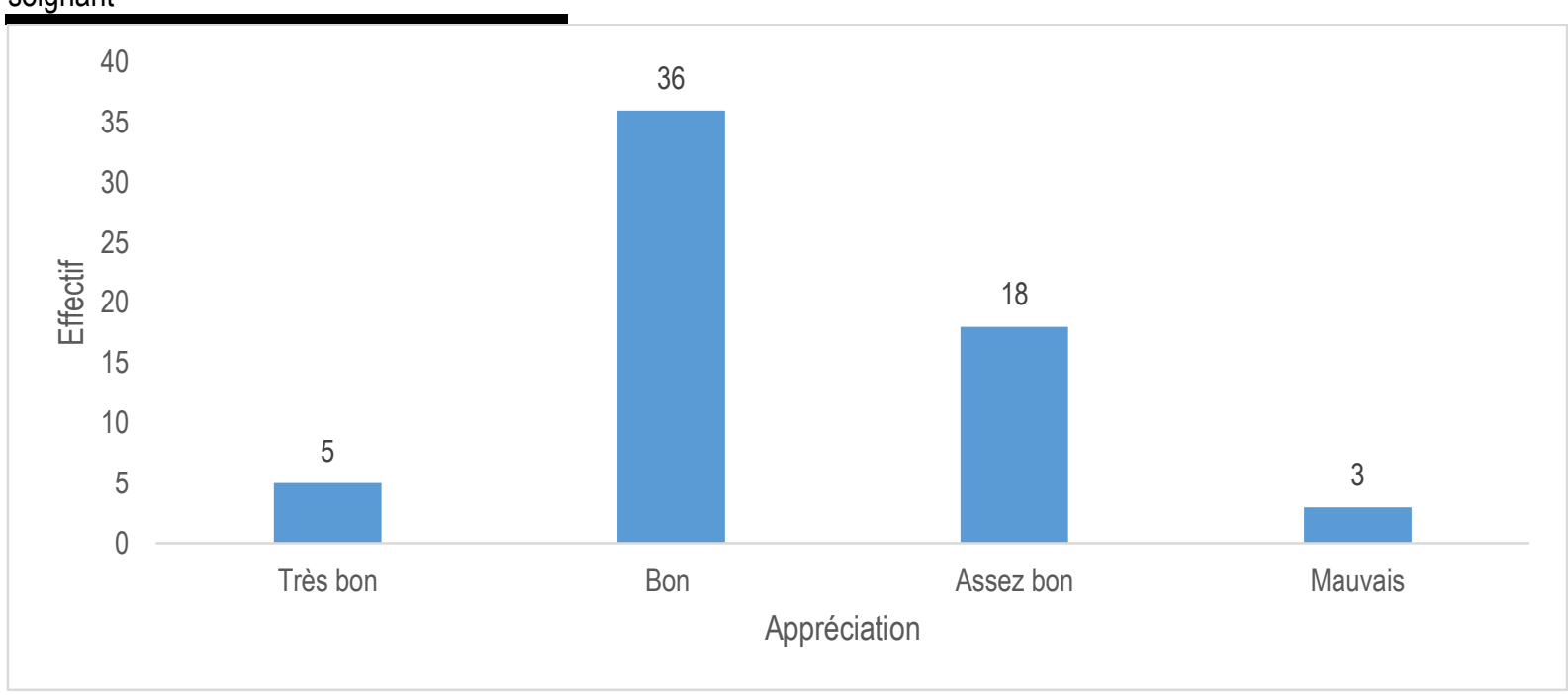

Graphique 1 : Appréciation de l'état de la salle d'hospitalisation par les des usagers

Tableau V : Répartition des usagers selon l'appréciation des coûts des examens paracliniques.

\begin{tabular}{lll}
\hline Appréciation & Effectif & $\%$ \\
\hline Très cher & 36 & $\mathbf{4 5 , 0 0}$ \\
Cher & 18 & 22,50 \\
Abordable & 17 & 21,25 \\
Moins cher & 3 & 3,75 \\
Ne sait pas & 6 & 7,50 \\
Total & 80 & 100,00 \\
\hline
\end{tabular}

Tableau VI : Statut matrimonial des usagers et appréciation de la satisfaction globale $p=0,009$

\begin{tabular}{llll}
\hline & \multicolumn{2}{c}{ Appréciation de la satisfaction globale } \\
Statut matrimonial & Bonne & Mauvaise & Total \\
\hline Célibataire & $5(6,25)$ & $5(6,25)$ & $10(12,50)$ \\
Marié(e) & $53(66,25)$ & $6(7,50)$ & $59(73,75)$ \\
Veuf(ve) & $6(7,50)$ & $3(3,75)$ & $9(11,25)$ \\
Divorcé(e) & $2(2,50)$ & $0(0,00)$ & $2(2,50)$ \\
Total & $66(82,50)$ & $14(17,50)$ & $80(100,00)$ \\
\hline
\end{tabular}


Tableau VII : Etat de la salle d'hospitalisation par rapport à la propreté et appréciation globale de la satisfaction des usagers

\begin{tabular}{|c|c|c|c|}
\hline \multicolumn{4}{|c|}{ Appréciation de la satisfaction globale } \\
\hline Propreté de la salle & Bonne & Mauvaise & Total \\
\hline Très bonne & $8(12,9)$ & $0(0,00)$ & $8(12,9)$ \\
\hline Bonne & $19(30,6)$ & $1(1,6)$ & $20(32,2)$ \\
\hline Assez-bonne & $22(35,6)$ & $8(12,9)$ & $30(48,5)$ \\
\hline Mauvaise & $2(3,2)$ & $2(3,2)$ & $4(6,4)$ \\
\hline Total & $51(82,3)$ & $11(17,7)$ & $62(100)$ \\
\hline
\end{tabular}

$p=0,038$

Tableau VIII : Appréciation de l'état des matelas par les usagers

\begin{tabular}{llll}
\hline & \multicolumn{3}{c}{ Appréciation de la satisfaction globale } \\
Etat des matelas & Bonne & Mauvaise & Total \\
\hline Confortable & $\mathbf{2 7}(\mathbf{4 3 , 5 )}$ & $4(6,5)$ & $\mathbf{3 1}(\mathbf{5 0 , 0 )}$ \\
Assez-confortable & $21(33,9)$ & $2(3,2)$ & $23(37,1)$ \\
Mauvais & $3(4,9)$ & $5(8,0)$ & $8(12,9)$ \\
Total & $51(82,3)$ & $11(17,7)$ & $62(100)$ \\
\hline $\mathrm{p}=0,002$ & & &
\end{tabular}

$p=0,002$ 